\title{
Palladium-Catalyzed Alcoholysis of 3-lodopropynamides: Selective Synthesis of Carbamoylacetates
}

\author{
Jian-Sheng Tang ${ }^{a, b}$ \\ Can-Cheng Guo*a \\ a College of Chemistry and Chemical Engineering, Hunan Uni- \\ versity, Changsha 410082, P. R. of China \\ ccguo@hnu.edu.cn \\ b Laboratory of Organic Chemistry, Hunan First Normal Univer- \\ sity, Changsha 410205, P. R. of China
}

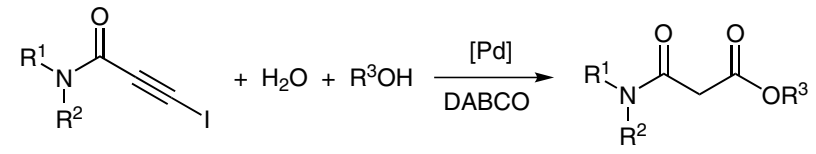

$\mathrm{R}^{1}=$ aryl, $\mathrm{Bn} \quad \mathrm{R}^{2}=\mathrm{Me}, \mathrm{H}, \mathrm{Ph} \quad \mathrm{R}^{3}=$ alkyl
Received: 10.07.2014

Accepted after revision: 15.08.2014

Published online: 25.09 .2014

DOI: 10.1055/s-0034-1379104; Art ID: ss-2014-h0439-op

Abstract A novel and selective method for the synthesis of carbamoylacetates via the alcoholysis of 3-iodopropynamides has been developed. 3-lodopropynamides react with alcohols in the presence of palladium(II) acetate and DABCO to afford the corresponding carbamoylacetates in moderate to good yields.

Key words palladium, 1,4-diazabicyclo[2.2.2]octane, alcoholysis, 3iodopropynamides, carbamoylacetates

Carbamoylacetates are versatile synthetic building blocks that are used for the synthesis of many natural products and related compounds of biological and medicinal importance. ${ }^{1}$ The traditional synthetic approach to carbamoylacetates is direct condensation of anilines with monoethyl malonates or alkyl malonyl chlorides. A few alternative methods, such as palladium-catalyzed carbonylation of diazo compounds with carbon monoxide, ${ }^{2}$ palladium/lightcatalyzed carbonylation of $\alpha$-iodoacetates, carbon monoxide, and amines, ${ }^{3}$ and other reactions, ${ }^{4}$ have been reported. Alkynyl iodides are a class of important compounds that have been used widely in organic synthesis. ${ }^{5}$ Recently, we also reported a modified protocol for the synthesis of internal alkynes, such as N,3-diarylpropynamides, by the palladium(II) acetate catalyzed Suzuki-Miyaura cross-coupling reaction of alkynyl iodides with arylboronic acids. ${ }^{5 f}$ Interestingly, however, in a similar reaction using 1,4-diazabicyclo[2.2.2] octane (DABCO) as base, we found that the envisioned internal alkyne was obtained only in low yield, and the carbamoylacetate was formed as the principal product. This led us to investigate the alcoholysis reaction, and here we present an efficient palladium and DABCO system catalyzed alcoholysis of 3-iodopropynamides to carbamoylacetates under an air atmosphere (Scheme 1).

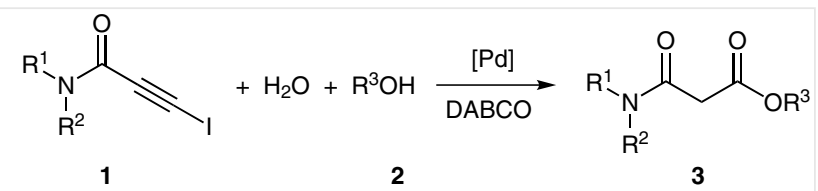

$\mathrm{R}^{1}=$ aryl, $\mathrm{Bn} \quad \mathrm{R}^{2}=\mathrm{Me}, \mathrm{H}, \mathrm{Ph} \quad \mathrm{R}^{3}=$ alkyl

Scheme 1 Palladium-catalyzed synthesis of carbamoylacetates

The reaction between 3-iodo- $N$-methyl- $N$-phenylpropynamide (1a) and methanol (2a) was chosen as a model reaction to screen the optimal reaction conditions; the results are summarized in Table 1. Initially, the effects of varying the palladium catalyst were examined. The results showed that treatment of 1a with 2 a using $2.0 \mathrm{~mol} \%$ palladium catalyst and two equivalents of DABCO in acetonitrile under an air atmosphere for 12 hours afforded the desired product 3 in 52, 71,62, and 83\% yields, respectively (entries 1-4). Palladium(II) acetate was the best catalyst in terms of yield, and the amount of palladium(II) acetate also affected the yield to some extent (entries 4-7). A variety of other bases, such as triethylamine, 4-(dimethylamino)pyridine, potassium tert-butoxide, and 1,8-diazabicyclo[5.4.0]undec7-ene (DBU) were then investigated, and they were inferior to DABCO (entries 4 and 8-11). It was noted that the reaction does not take place with cesium carbonate (entry 12). Finally, the use of various solvents, including methanol, tetrahydrofuran, $\mathrm{N}, \mathrm{N}$-dimethylformamide, 1,2-dichloroethane, and toluene, was also examined, and acetonitrile provided the highest yield (entries 4 and 13-17); methanol gave identical results to acetonitrile (entry 13). In view of the high-boiling or complex alcohols which were not easily purified, acetonitrile was chosen over methanol as a solvent. 
Table 1 Screening Optimal Conditions ${ }^{\mathrm{a}}$

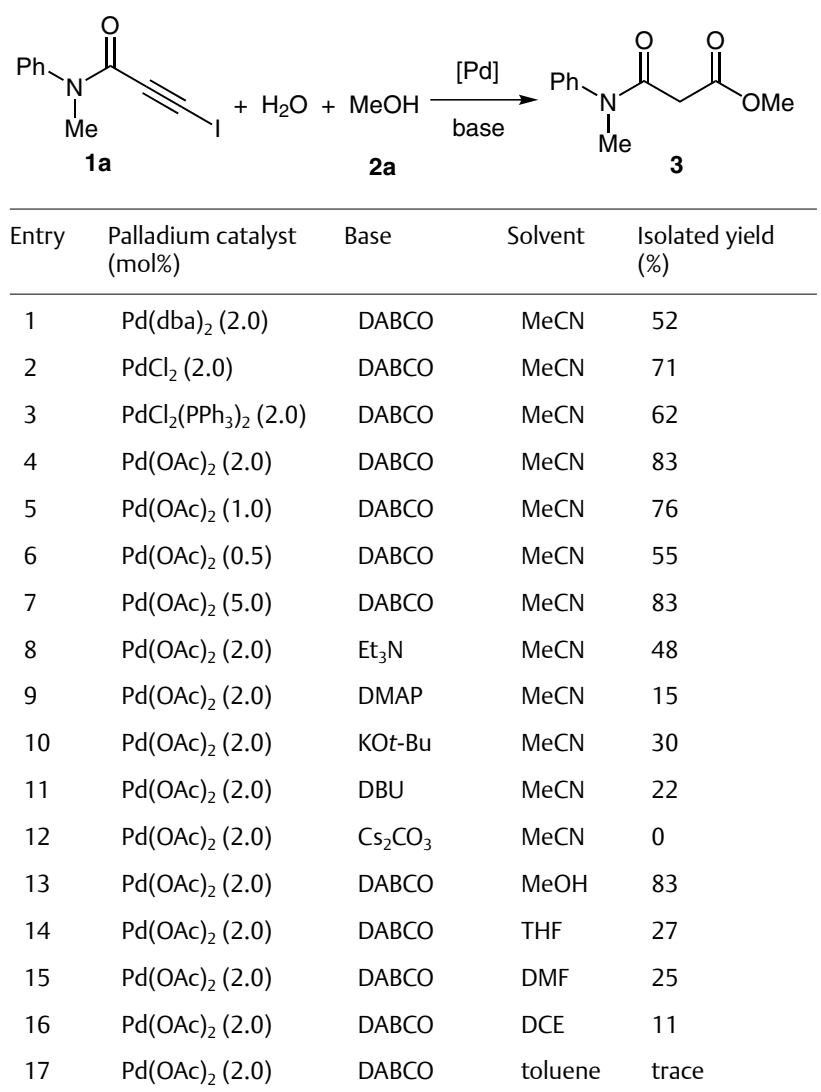

a Reaction conditions: 1 a $(0.2 \mathrm{mmol}), 2 \mathrm{a}(0.4 \mathrm{mmol})$, base (2 equiv), solvent $(2 \mathrm{~mL})$, r.t., $12 \mathrm{~h}$, air atmosphere.

With the optimal reaction conditions in hand, the scope of the 3-iodopropynamide and alcohol was investigated (Table 2). Initially, we turned our attention to examine suitable alcohols for the reaction. The results demonstrated that a variety of alcohols $\mathbf{2 b}-\mathbf{e}$ all worked well with 3-iodo$\mathrm{N}$-methyl- $\mathrm{N}$-phenylpropynamide (1a) in moderate to good yields (entries 1-4). For example, reaction of $\mathbf{2 b}$ or $\mathbf{2} \mathbf{c}$ with 1a in the presence of $2.0 \mathrm{~mol} \%$ palladium catalyst and two equivalents of DABCO in acetonitrile under an air atmosphere for 12 hours gave the corresponding carbamoylacetates $\mathbf{4}$ and $\mathbf{5}$ in 83 and 75\% yields, respectively (entries 1 and 2). The addition of benzyl alcohol afforded the product 6 in good yield (entry 3). Gratifyingly, the sterically hindered tertiary alcohol $\mathbf{2 e}$ was also consistent with the reaction conditions, and gave the desired product $\mathbf{7}$ in moderate yield (entry 4). Subsequently, a number of 3-iodopropynamides $\mathbf{1 b}$-h were examined in the presence of the alcohol, palladium(II) acetate, and DABCO (entries 5-11). We found that reaction of substituted phenyl 3-iodopropynamides $\mathbf{1 b}$ and $\mathbf{1 c}$ with ethanol (2b) afforded the corresponding products 8 and 9 in 81 and 78\% yields, respectively (entries 5 and 6). Comparing $N$-phenyl- with $N$-(o-tolyl)and $\mathrm{N}$-( $\mathrm{m}$-tolyl)propynamides, meta substitution on the phenyl ring was found to have some effect on the reaction, and the yield of $\mathbf{1 2}$ slightly decreased to $60 \%$ compared to 71-73\% for 10 and 11 (entries 7-9). Although the activity was reduced for the reaction, 3-iodo- $N, N$-diphenylpropynamide (19) and $\mathrm{N}$-benzyl-3-iodo- $\mathrm{N}$-methylpropynamide ( $\mathbf{1 h})$ were also suitable substrates leading to the desired products 13 and $\mathbf{1 4}$ smoothly in moderate yields (entries 10 and 11). Surprisingly, the reaction of phenyl 3-

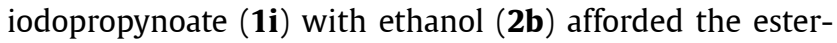
exchanged product, diethyl malonate (15), in $42 \%$ yield (entry 12 ). However, no product was generated using 3-iodo$\mathrm{N}$-methyl- $\mathrm{N}$-phenylpropynamide (1a) and diethylamine (2f) under the optimal reaction conditions.

The products of alcoholysis of 3-iodopropynamides provide an attractive and useful route to introduce new groups in the synthesis of natural products. For example, methyl 3[methyl(phenyl)amino]-3-oxopropanoate (3) underwent a methylation-cyclization route to give an oxoindoline derivative $\mathbf{1 6}$ in good yield (Scheme 2). ${ }^{6}$

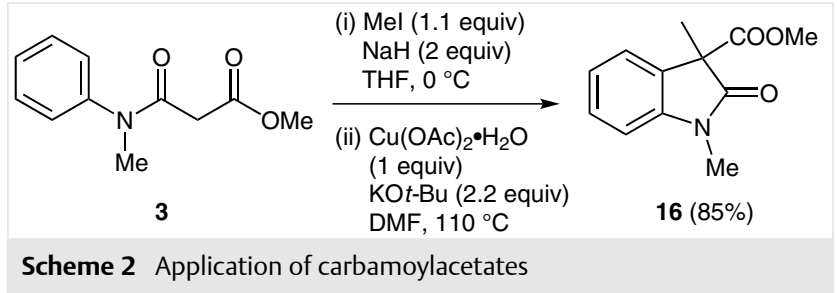

In summary, we have developed a novel, efficient and facile protocol for the synthesis of carbamoylacetates by the palladium(II) acetate/DABCO system catalyzed alcoholysis of 3-iodopropynamides under aerobic conditions. This protocol is simple and highly general for the diverse substrate scope, which provides a valuable complement to traditional methods. Further utilization of this procedure will continue in our laboratory.

Chemicals were purchased from commercial supplier (Aldrich, USA, and Changsha Chemical Company, China) and used without purification prior to use. NMR spectroscopy was performed on a Bruker-500 spectrometer operating at $500 \mathrm{MHz}\left({ }^{1} \mathrm{H}\right.$ NMR $)$ and $125 \mathrm{MHz}\left({ }^{13} \mathrm{C}\right.$ NMR). TMS was used an internal standard and $\mathrm{CDCl}_{3}$ was used as the solvent. HRMS data were performed on a micro-TOF mass spectrometer

\section{3-Iodo- $N$-methyl- $N$-phenylpropynamide (1a); Typical Procedure}

To a solution of propynoic acid $(154.0 \mathrm{mg}, 2.2 \mathrm{mmol})$ and $\mathrm{N}$-methylaniline $(214.0 \mathrm{mg}, 2 \mathrm{mmol})$ in $\mathrm{CH}_{2} \mathrm{Cl}_{2}(6 \mathrm{~mL})$ was added gradually a solution of DCC ( $453.2 \mathrm{mg}, 2.2 \mathrm{mmol})$ in $\mathrm{CH}_{2} \mathrm{Cl}_{2}(6 \mathrm{~mL})$ at $0{ }^{\circ} \mathrm{C}$, then the mixture was stirred at r.t. for $1 \mathrm{~h}$. After purification, the product was then treated with NIS (495 mg, $2.2 \mathrm{mmol}$ ) and $\mathrm{AgNO}_{3}(34.0 \mathrm{mg}$, $0.2 \mathrm{mmol}$ ) in acetone at r.t. under an air atmosphere for $8 \mathrm{~h}$ until complete consumption of the starting material (TLC and GC analysis). ${ }^{7}$ When the reaction was finished, sat. $\mathrm{Na}_{2} \mathrm{~S}_{2} \mathrm{O}_{3}(10 \mathrm{~mL})$ was added to the mixture, and then aqueous phase was extracted with $\mathrm{Et}_{2} \mathrm{O}$. The combined organic extracts were dried (anhyd $\mathrm{Na}_{2} \mathrm{SO}_{4}$ ), and evaporated in 
Table 2 Alcoholysis of 3-lodopropynamide $\mathbf{1}^{\mathrm{a}}$

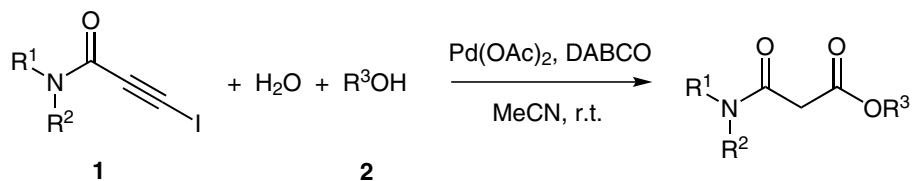

\begin{tabular}{|c|c|c|c|c|c|c|c|}
\hline Entry & Propynamide & $\mathrm{R}^{1}$ & $\mathrm{R}^{2}$ & Alcohol & $\mathrm{R}^{3}$ & Product & Yield ${ }^{\mathrm{b}}(\%)$ \\
\hline 1 & $1 a$ & $\mathrm{Ph}$ & $\mathrm{Me}$ & $2 b$ & Et & 4 & 83 \\
\hline 2 & $1 a$ & $\mathrm{Ph}$ & Me & $2 c$ & $\mathrm{CH}_{2} \mathrm{CH}=\mathrm{CH}_{2}$ & 5 & 75 \\
\hline 3 & $1 a$ & $\mathrm{Ph}$ & $\mathrm{Me}$ & $2 d$ & $\mathrm{Bn}$ & 6 & 80 \\
\hline 4 & $1 a$ & $\mathrm{Ph}$ & Me & $2 e$ & $t-B u$ & 7 & 66 \\
\hline 5 & $1 b$ & 4- $\mathrm{MeC}_{6} \mathrm{H}_{4}$ & $\mathrm{Me}$ & $2 b$ & Et & 8 & 81 \\
\hline 6 & $1 c$ & $4-\mathrm{ClC}_{6} \mathrm{H}_{4}$ & Me & $2 b$ & Et & 9 & 78 \\
\hline 7 & 1d & $\mathrm{Ph}$ & $\mathrm{H}$ & $2 a$ & Me & 10 & 71 \\
\hline 8 & $1 e$ & $2-\mathrm{MeC}_{6} \mathrm{H}_{4}$ & $\mathrm{H}$ & $2 a$ & Me & 11 & 73 \\
\hline 9 & $1 f$ & $3-\mathrm{MeC}_{6} \mathrm{H}_{4}$ & $\mathrm{H}$ & $2 a$ & Me & 12 & 60 \\
\hline 10 & $1 \mathrm{~g}$ & $\mathrm{Ph}$ & $\mathrm{Ph}$ & $2 a$ & Me & 13 & 55 \\
\hline 11 & $1 \mathrm{~h}$ & $\mathrm{Bn}$ & $\mathrm{Me}$ & $2 a$ & Me & 14 & 48 \\
\hline $12^{\mathrm{c}}$ & $1 \mathrm{i}$ & $--^{d}$ & $-{ }^{d}$ & $2 b$ & Et & 15 & 42 \\
\hline 13 & $1 a$ & $\mathrm{Ph}$ & $\mathrm{Me}$ & $2 f$ & -e $^{\mathrm{e}}$ & $-^{f}$ & $-^{f}$ \\
\hline
\end{tabular}

${ }^{a}$ Reaction conditions: 1 ( $\left.0.2 \mathrm{mmol}\right), 2$ (2 equiv), DABCO (2 equiv), MeCN ( $2 \mathrm{~mL}$ ), r.t., air atmosphere, $12 \mathrm{~h}$.

${ }^{\mathrm{b}}$ Isolated yield.

c Diethyl malonate (15) was obtained.

d Substrate was phenyl 3-iodopropynoate.

e Diethylamine was used.

${ }^{\mathrm{f}}$ No reaction.

vacuo, the residue was purified by flash column chromatography (silica gel, hexane-EtOAc) to give the corresponding 3-iodo- $N$-methyl- $N$ phenylpropynamide (1a).

Substrates $\mathbf{1}$ were synthesized according to the typical procedure. Substrates 1a,b,d,g,i are known; $\mathbf{1 c , e , f , h , ~ w i t h ~ s t r u c t u r e s ~ s i m i l a r ~ t o ~}$ the known compounds, were confirmed by GC-MS and used directly for the alcoholysis reaction.

\section{$\mathbf{N}$-(4-Chlorophenyl)-3-iodo- $\mathbf{N}$-methylpropiolamide (1c)}

MS (EI, $70 \mathrm{eV}): m / z(\%)=319(64)[\mathrm{M}]^{+}, 291$ (6), 262 (32), 140 (100), 111 (40).

\section{3-Iodo- $N$-(2-tolyl)propiolamide (1e)}

MS (EI, $70 \mathrm{eV}): m / z(\%)=285(80)[\mathrm{M}]^{+}, 257$ (18), 179 (100), 158 (55), 130 (76).

\section{3-Iodo- $\mathbf{N}$-(3-tolyl)propiolamide (1f)}

MS (EI, $70 \mathrm{eV}): m / z(\%)=285(76)[\mathrm{M}]^{+}, 257$ (16), 179 (100), 158 (60), 130 (72).

\section{$\mathbf{N}$-Benzyl-3-iodo- $\mathbf{N}$-methylpropiolamide (1h)}

MS (EI, $70 \mathrm{eV}): m / z(\%)=299(85)[\mathrm{M}]^{+}, 271(18), 242(28), 120$ (100).

\section{Carbamoylacetates 3-15; General Procedure}

A mixture of 3-iodopropynamide 1 (0.2 mmol), alcohol 2 (0.4 mmol), $\mathrm{Pd}(\mathrm{OAc})_{2}$ (0.896 mg, $\left.0.004 \mathrm{mmol}\right)$, and DABCO (44.8 $\left.\mathrm{mg}, 0.4 \mathrm{mmol}\right)$ was stirred in $\mathrm{MeCN}(2 \mathrm{~mL}$ ) at r.t. for $12 \mathrm{~h}$ until complete consumption of the starting material (TLC and GC analysis). When the reaction was complete, sat. $\mathrm{Na}_{2} \mathrm{~S}_{2} \mathrm{O}_{3}(10 \mathrm{ml})$ was added to the mixture and it was extracted with $\mathrm{Et}_{2} \mathrm{O}(3 \times 20 \mathrm{~mL})$. The combined organic extracts were dried (anhyd $\mathrm{Na}_{2} \mathrm{SO}_{4}$ ) and evaporated in vacuo and the residue was purified by flash column chromatography (silica gel, EtOAc-hexane) to afford the desired product (Table 2).

\section{Methyl 3-[Methyl(phenyl)amino]-3-oxopropanoate (3) 1e $^{1 \mathrm{e}}$}

Colorless oil; yield: $34.4 \mathrm{mg}$ (83\%).

IR (KBr): 1747, $1651 \mathrm{~cm}^{-1}$.

${ }^{1} \mathrm{H}$ NMR (500 MHz, CDCl $)$ : $\delta=7.45-7.42(\mathrm{~m}, 2 \mathrm{H}), 7.38(\mathrm{~d}, J=7.0 \mathrm{~Hz}, 1$ H), 7.24-7.23 (m, $2 \mathrm{H}), 3.68$ (s, $3 \mathrm{H}), 3.31(\mathrm{~s}, 3 \mathrm{H}), 3.23$ (s, $2 \mathrm{H})$.

${ }^{13} \mathrm{C} \mathrm{NMR}\left(125 \mathrm{MHz}, \mathrm{CDCl}_{3}\right): \delta=168.1,165.9,143.4,129.9,128.3$, 127.2, 52.2, 41.3, 37.4 .

HRMS (EI): $m / z$ [M] $]^{+}$calcd for $\mathrm{C}_{11} \mathrm{H}_{13} \mathrm{NO}_{3}$ : 207.0895; found: 207.0889 .

Ethyl 3-[Methyl(phenyl)amino]-3-oxopropanoate (4) ${ }^{1 \mathrm{e}}$

Slightly yellow oil; yield: $36.7 \mathrm{mg}$ (83\%).

IR (KBr): 1740, $1666 \mathrm{~cm}^{-1}$.

${ }^{1} \mathrm{H}$ NMR (500 MHz, $\mathrm{CDCl}_{3}$ ): $\delta=7.43(\mathrm{t}, J=7.5 \mathrm{~Hz}, 2 \mathrm{H}), 7.37$ (d, $J=7.0$ $\mathrm{Hz}, 1 \mathrm{H}), 7.24(\mathrm{~d}, J=7.5 \mathrm{~Hz}, 2 \mathrm{H}), 4.15-4.11(\mathrm{~m}, 2 \mathrm{H}), 3.31(\mathrm{~s}, 3 \mathrm{H}), 3.21$ $(\mathrm{s}, 2 \mathrm{H}), 1.25(\mathrm{t}, J=7.5 \mathrm{~Hz}, 3 \mathrm{H})$.

${ }^{13} \mathrm{C}$ NMR $\left(125 \mathrm{MHz}, \mathrm{CDCl}_{3}\right): \delta=167.8,166.1,143.5,130.0,128.3$, 127.3, 61.3, 41.6, 37.5, 14.1.

HRMS (EI): $m / z$ [M] $]^{+}$calcd for $\mathrm{C}_{12} \mathrm{H}_{15} \mathrm{NO}_{3}$ : 221.1052; found: 221.1048 . 
Allyl 3-[Methyl(phenyl)amino]-3-oxopropanoate (5)

Pale yellow oil; yield: $35.0 \mathrm{mg}$ (75\%).

IR (KBr): 1744, $1667 \mathrm{~cm}^{-1}$.

${ }^{1} \mathrm{H}$ NMR $\left(500 \mathrm{MHz}, \mathrm{CDCl}_{3}\right): \delta=7.44(\mathrm{t}, J=8.0 \mathrm{~Hz}, 2 \mathrm{H}), 7.37(\mathrm{~d}, J=9.0$ $\mathrm{Hz}, 1 \mathrm{H}), 7.25(\mathrm{t}, J=10.5 \mathrm{~Hz}, 2 \mathrm{H}), 5.92-5.83(\mathrm{~m}, 1 \mathrm{H}), 5.32-5.21(\mathrm{~m}, 2$ $\mathrm{H}), 4.57(\mathrm{~d}, J=7.0 \mathrm{~Hz}, 2 \mathrm{H}), 3.31(\mathrm{~s}, 3 \mathrm{H}), 3.25(\mathrm{~s}, 2 \mathrm{H})$.

${ }^{13} \mathrm{C}$ NMR $\left(125 \mathrm{MHz}, \mathrm{CDCl}_{3}\right): \delta=167.4,165.9,143.5,131.7,130.0$, $128.3,127.3,118.6,65.8,41.5,37.5$.

HRMS (EI): $m / z$ [M] $]^{+}$calcd for $\mathrm{C}_{13} \mathrm{H}_{15} \mathrm{NO}_{3}$ : 233.1052; found: 233.1047.

\section{Benzyl 3-[Methyl(phenyl)amino]-3-oxopropanoate (6) ${ }^{1 \mathrm{f}}$}

Colorless oil; yield: $45.3 \mathrm{mg}(80 \%)$.

IR (KBr): 1746, $1660 \mathrm{~cm}^{-1}$.

${ }^{1} \mathrm{H}$ NMR $\left(500 \mathrm{MHz}, \mathrm{CDCl}_{3}\right): \delta=7.38-7.31(\mathrm{~m}, 8 \mathrm{H}), 7.20-7.17(\mathrm{~m}, 2 \mathrm{H})$, $5.12(\mathrm{~s}, 2 \mathrm{H}), 3.31$ (s, $3 \mathrm{H}), 3.27$ (s, $2 \mathrm{H})$.

${ }^{13} \mathrm{C}$ NMR $\left(125 \mathrm{MHz}, \mathrm{CDCl}_{3}\right): \delta=167.6,165.8,143.4,135.5,130.0$, $128.5,128.4,128.3,128.3,127.2,67.0,41.6,37.5$.

HRMS (EI): $m / z$ [M] $]^{+}$calcd for $\mathrm{C}_{17} \mathrm{H}_{17} \mathrm{NO}_{3}$ : 283.1208; found: 283.1202 .

\section{tert-Butyl 3-[Methyl(phenyl)amino]-3-oxopropanoate (7)}

Colorless oil; yield: $32.9 \mathrm{mg}$ (66\%).

IR (KBr): 1735, $1664 \mathrm{~cm}^{-1}$.

${ }^{1} \mathrm{H}$ NMR (500 MHz, $\left.\mathrm{CDCl}_{3}\right): \delta=7.44-7.41(\mathrm{~m}, 2 \mathrm{H}), 7.37(\mathrm{~d}, J=7.0 \mathrm{~Hz}, 1$ H), $7.23(\mathrm{t}, J=6.5 \mathrm{~Hz}, 2 \mathrm{H}), 3.30(\mathrm{~s}, 3 \mathrm{H}), 3.18(\mathrm{~s}, 2 \mathrm{H}), 1.41(\mathrm{~s}, 9 \mathrm{H})$.

${ }^{13} \mathrm{C}$ NMR $\left(125 \mathrm{MHz}, \mathrm{CDCl}_{3}\right): \delta=167.0,166.4,143.7,129.8,128.1$, $127.3,81.5,42.7,37.4,28.0$.

HRMS (EI): $m / z$ [M] $]^{+}$calcd for $\mathrm{C}_{14} \mathrm{H}_{19} \mathrm{NO}_{3}$ : 249.1365; found: 249.1359 .

\section{Ethyl 3-[Methyl(p-tolyl)amino]-3-oxopropanoate $(8)^{1 \mathrm{~d}}$}

Colorless oil; yield: $38.1 \mathrm{mg}(81 \%)$.

IR (KBr): $1741,1667 \mathrm{~cm}^{-1}$.

${ }^{1} \mathrm{H}$ NMR (500 MHz, $\left.\mathrm{CDCl}_{3}\right)$ : $\delta=7.21(\mathrm{~d}, J=12.0 \mathrm{~Hz}, 2 \mathrm{H}), 7.11(\mathrm{~d}, J=5.5$ $\mathrm{Hz}, 2 \mathrm{H}$ ), 4.15-4.10 (m, $2 \mathrm{H}), 3.28$ (s, $3 \mathrm{H}), 3.21$ (s, $2 \mathrm{H}), 2.38$ (s, $3 \mathrm{H})$, $1.23(\mathrm{t}, J=8.5 \mathrm{~Hz}, 3 \mathrm{H})$.

${ }^{13} \mathrm{C}$ NMR $\left(125 \mathrm{MHz}, \mathrm{CDCl}_{3}\right): \delta=167.8,166.2,141.0,138.2,130.5$, 127.0, 61.2, 41.5, 37.4, 21.1, 14.1.

HRMS (EI): $m / z$ [M] $]^{+}$calcd for $\mathrm{C}_{13} \mathrm{H}_{17} \mathrm{NO}_{3}$ : 235.1208; found: 235.1203 .

\section{Ethyl 3-[4-Chlorophenyl(methyl)amino]-3-oxopropanoate (9) ${ }^{1 \mathrm{~d}}$}

White solid; yield: $39.8 \mathrm{mg}$ (78\%); $\mathrm{mp} 49-50{ }^{\circ} \mathrm{C}$.

IR (KBr): $1741,1666 \mathrm{~cm}^{-1}$.

${ }^{1} \mathrm{H}$ NMR (500 MHz, $\mathrm{CDCl}_{3}$ ): $\delta=7.40(\mathrm{~d}, J=10.5 \mathrm{~Hz}, 2 \mathrm{H}), 7.19$ (d, $J=$ $10.5 \mathrm{~Hz}, 2 \mathrm{H}), 4.16-4.10(\mathrm{~m}, 2 \mathrm{H}), 3.29(\mathrm{~s}, 3 \mathrm{H}), 3.20(\mathrm{~s}, 2 \mathrm{H}), 1.24(\mathrm{t}, J=$ $9.0 \mathrm{~Hz}, 3 \mathrm{H})$.

${ }^{13} \mathrm{C}$ NMR $\left(125 \mathrm{MHz}, \mathrm{CDCl}_{3}\right): \delta=167.8,166.2,142.0,134.1,130.1$, 128.7, 61.3, 41.4, 37.4, 14.0.

HRMS (EI): $m / z[M]^{+}$calcd for $\mathrm{C}_{12} \mathrm{H}_{14} \mathrm{ClNO}_{3}$ : 255.0662; found: 255.0658 .

\section{Methyl 3-0xo-3-(phenylamino)propanoate $(10)^{4 a}$}

Brown solid; yield: $27.4 \mathrm{mg}(71 \%) ; \mathrm{mp} 42-44^{\circ} \mathrm{C}$.

IR (KBr): $1740,1670 \mathrm{~cm}^{-1}$.
${ }^{1} \mathrm{H}$ NMR $\left(500 \mathrm{MHz}, \mathrm{CDCl}_{3}\right): \delta=9.25(\mathrm{~s}, 1 \mathrm{H}), 7.53(\mathrm{~d}, J=8.0 \mathrm{~Hz}, 2 \mathrm{H})$, $7.29(\mathrm{t}, J=8.0 \mathrm{~Hz}, 2 \mathrm{H}), 7.10(\mathrm{t}, J=7.5 \mathrm{~Hz}, 1 \mathrm{H}), 3.75(\mathrm{~s}, 3 \mathrm{H}), 3.46(\mathrm{~s}, 2$ $\mathrm{H})$.

${ }^{13} \mathrm{C}$ NMR $\left(125 \mathrm{MHz}, \mathrm{CDCl}_{3}\right): \delta=169.6,163.3,137.4,128.8,124.5$, 120.0, 52.4, 41.8 .

HRMS (EI): $m / z$ [M] calcd for $\mathrm{C}_{10} \mathrm{H}_{11} \mathrm{NO}_{3}$ : 193.0739; found: 193.0734 .

\section{Methyl 3-0xo-3-(o-tolylamino)propanoate (11)}

Colorless oil; yield: $30.2 \mathrm{mg}$ (73\%).

IR (KBr): $1741,1668 \mathrm{~cm}^{-1}$.

${ }^{1} \mathrm{H} \mathrm{NMR}\left(500 \mathrm{MHz}, \mathrm{CDCl}_{3}\right): \delta=9.23(\mathrm{~s}, 1 \mathrm{H}), 7.96(\mathrm{~d}, J=8.0 \mathrm{~Hz}, 1 \mathrm{H})$, 7.22-7.19 (m, $2 \mathrm{H}), 7.07$ (t, J = 7.5 Hz, $1 \mathrm{H}), 3.82(\mathrm{~s}, 3 \mathrm{H}), 3.53(\mathrm{~s}, 2 \mathrm{H})$, $2.33(\mathrm{~s}, 3 \mathrm{H})$.

${ }^{13} \mathrm{C}$ NMR $\left(125 \mathrm{MHz}, \mathrm{CDCl}_{3}\right): \delta=170.8,162.8,135.6,130.4,128.5$, 126.7, 125.0, 122.2, 52.6, 41.0, 17.8.

HRMS (EI): $m / z$ [M] $]^{+}$calcd for $\mathrm{C}_{11} \mathrm{H}_{13} \mathrm{NO}_{3}$ : 207.0895; found: 207.0889 .

\section{Methyl 3-0xo-3-(m-tolylamino)propanoate (12) ${ }^{9}$}

Pale yellow solid; yield: $24.8 \mathrm{mg}(60 \%)$; $\mathrm{mp} 38-40{ }^{\circ} \mathrm{C}$.

IR (KBr): 1742, $1666 \mathrm{~cm}^{-1}$.

${ }^{1} \mathrm{H}$ NMR $\left(500 \mathrm{MHz}, \mathrm{CDCl}_{3}\right): \delta=9.10(\mathrm{~s}, 1 \mathrm{H}), 7.38(\mathrm{~s}, 1 \mathrm{H}), 7.34(\mathrm{~d}, J=$ $8.0 \mathrm{~Hz}, 1 \mathrm{H}), 7.20(\mathrm{t}, J=7.5 \mathrm{~Hz}, 1 \mathrm{H}), 6.94(\mathrm{~d}, J=7.0 \mathrm{~Hz}, 1 \mathrm{H}), 3.79(\mathrm{~s}, 3$ $\mathrm{H}), 3.47(\mathrm{~s}, 2 \mathrm{H}), 2.33(\mathrm{~s}, 3 \mathrm{H})$.

${ }^{13} \mathrm{C}$ NMR $\left(125 \mathrm{MHz}, \mathrm{CDCl}_{3}\right): \delta=170.2,162.8,138.9,137.3,128.8$, $125.4,120.7,117.2,52.6,41.5,21.4$.

HRMS (EI): $m / z$ [M] $]^{+}$calcd for $\mathrm{C}_{11} \mathrm{H}_{13} \mathrm{NO}_{3}$ : 207.0895; found: 207.0888 .

Methyl 3-(Diphenylamino)-3-oxopropanoate (13) ${ }^{10}$

White solid; yield: $29.6 \mathrm{mg}$ (55\%); $\mathrm{mp} 81-83^{\circ} \mathrm{C}$.

IR (KBr): $1734,1649 \mathrm{~cm}^{-1}$.

${ }^{1} \mathrm{H}$ NMR $\left(500 \mathrm{MHz}, \mathrm{CDCl}_{3}\right): \delta=7.33-7.21(\mathrm{~m}, 10 \mathrm{H}), 3.67(\mathrm{~s}, 3 \mathrm{H}), 3.41$ (s, $2 \mathrm{H})$.

${ }^{13} \mathrm{C}$ NMR $\left(125 \mathrm{MHz}, \mathrm{CDCl}_{3}\right): \delta=167.9,165.8,129.9,128.9,128.5$, $128.3,126.3,126.2,52.3,42.4$.

HRMS (EI): $m / z$ [M] $]^{+}$calcd for $\mathrm{C}_{16} \mathrm{H}_{15} \mathrm{NO}_{3}$ : 269.1052; found: 269.1047 .

\section{Methyl 3-[Benzyl(methyl)amino]-3-oxopropanoate (14) ${ }^{1 \mathrm{~b}}$}

Brown oil; yield: $21.2 \mathrm{mg}$ (48\%).

IR (KBr): $1745,1699 \mathrm{~cm}^{-1}$.

${ }^{1} \mathrm{H}$ NMR $\left(500 \mathrm{MHz}, \mathrm{CDCl}_{3}\right): \delta=7.33-7.31(\mathrm{~m}, 2 \mathrm{H}), 7.29-7.27(\mathrm{~m}, 3 \mathrm{H})$, 4.62 (s, $2 \mathrm{H}), 3.77$ (s, $3 \mathrm{H}), 3.54$ (s, $2 \mathrm{H}), 2.92(\mathrm{~s}, 3 \mathrm{H})$.

${ }^{13} \mathrm{C}$ NMR $\left(125 \mathrm{MHz}, \mathrm{CDCl}_{3}\right): \delta=168.0,166.4,136.7,128.7,127.9$, 126.4, 52.5, 50.9, 41.3, 35.3.

HRMS (EI): $m / z$ [M] $]^{+}$calcd for $\mathrm{C}_{12} \mathrm{H}_{15} \mathrm{NO}_{3}$ : 221.1052; found: 221.1046 .

Diethyl Malonate (15) ${ }^{11}$

Colorless oil; yield: $13.4 \mathrm{mg}$ (42\%).

IR (KBr): $1747,1733 \mathrm{~cm}^{-1}$.

${ }^{1} \mathrm{H}$ NMR $\left(500 \mathrm{MHz}, \mathrm{CDCl}_{3}\right): \delta=4.22-4.18(\mathrm{~m}, 4 \mathrm{H}), 3.37(\mathrm{~s}, 2 \mathrm{H}), 1.30$ $1.27(\mathrm{~m}, 6 \mathrm{H})$.

${ }^{13} \mathrm{C}$ NMR $\left(125 \mathrm{MHz}, \mathrm{CDCl}_{3}\right): \delta=166.4,61.2,41.4,13.8$.

MS (EI, $70 \mathrm{eV}): m / z(\%)=160(2)[\mathrm{M}]^{+}, 133(67), 115(100)$. 


\section{Methyl 1,3-Dimethyl-2-oxo-2,3-dihydro-1H-indole-3-carboxylate (16); Typical Procedure}

To a solution of methyl 3-[methyl(phenyl)amino]-3-oxopropanoate $(3,103.5 \mathrm{mg}, 0.5 \mathrm{mmol})$ and $\mathrm{NaH}(24 \mathrm{mg}, 1 \mathrm{mmol})$ in THF (4 mL) was added gradually MeI $(78.1 \mathrm{mg}, 0.55 \mathrm{mmol})$ at $0{ }^{\circ} \mathrm{C}$, then the mixture was stirred for $5 \mathrm{~min}$ to afford methyl 2-methyl-3-[methyl(phenyl)amino]-3-oxopropanoate. After purification, the product was used for the cyclization reaction.

A mixture of methyl 2-methyl-3-[methyl(phenyl)amino]-3-oxopropanoate (44.2 $\mathrm{mg}, 0.2 \mathrm{mmol}), \mathrm{Cu}(\mathrm{OAc})_{2} \cdot \mathrm{H}_{2} \mathrm{O}(39.9 \mathrm{mg}, 0.2 \mathrm{mmol})$, and $\mathrm{KOt}$ - Bu (49.3 mg, $0.44 \mathrm{mmol})$ in DMF $(3 \mathrm{~mL})$ was stirred at $110{ }^{\circ} \mathrm{C}$ for $1 \mathrm{~h}$. Then sat. $\mathrm{NH}_{4} \mathrm{Cl}$ solution $(10 \mathrm{~mL})$ was added to the mixture and the aqueous phase was extracted with $\mathrm{Et}_{2} \mathrm{O}$. The combined organic extracts were dried (anhyd $\mathrm{Na}_{2} \mathrm{SO}_{4}$ ) and evaporated under reduced pressure. The residue was purified by flash column chromatography (hexane-EtOAc) to afford the desired product $(\mathbf{1 6})^{6}(37.2 \mathrm{mg}, 85 \%)$ as a white solid; $\mathrm{mp} 83-85^{\circ} \mathrm{C}$.

IR (KBr): $1743,1611 \mathrm{~cm}^{-1}$.

${ }^{1} \mathrm{H}$ NMR (300 MHz, $\left.\mathrm{CDCl}_{3}\right): \delta=7.34(\mathrm{t}, J=7.5 \mathrm{~Hz}, 1 \mathrm{H}), 7.27-7.26(\mathrm{~m}, 1$ $\mathrm{H}), 7.08(\mathrm{t}, J=6.5 \mathrm{~Hz}, 1 \mathrm{H}), 6.87(\mathrm{~d}, J=6.5 \mathrm{~Hz}, 1 \mathrm{H}), 3.68(\mathrm{~s}, 3 \mathrm{H}), 3.28(\mathrm{~s}$, $3 \mathrm{H}), 1.70(\mathrm{~s}, 3 \mathrm{H})$.

${ }^{13} \mathrm{C} \mathrm{NMR}\left(75 \mathrm{MHz}, \mathrm{CDCl}_{3}\right): \delta=175.1,170.3,143.6,130.3,129.1,123.1$, 122.9, 108.5, 54.9, 53.0, 26.6, 20.2.

HRMS (EI): $m / z$ [M] calcd for $\mathrm{C}_{12} \mathrm{H}_{13} \mathrm{NO}_{3}$ : 219.0895; found: 219.0891 .

\section{Acknowledgment}

The authors thank the National Natural Science Foundation of China (No. 21372068) for financial support.

\section{Supporting Information}

Supporting information for this article is available online at http://dx.doi.org/10.1055/s-0034-1379104.

\section{References}

(1) For reviews, see: (a) Pugh, D. S.; Klein, J. E. M. N.; Perry, A.; Taylor, R. J. K. Synlett 2010, 934. (b) Adediran, S. A.; Cabaret, D.; Lohier, J.-F.; Wakselman, M.; Pratt, R. F. Bioorg. Med. Chem. 2010, 18, 282. (c) Russo, A.; Galdi, G.; Croce, G.; Lattanzia, A. Chem. Eur. J. 2012, 18, 6152. (d) Wang, J.; Yuan, Y.; Xiong, R.; Zhang-Negrerie, D.; Du, Y.; Zhao, K. Org. Lett. 2012, 14, 2210. (e) Ghosh, S.; De, S.; Kakde, B. N.; Bhunia, S.; Adhikary, A.; Bisai, A. Org. Lett. 2012, 14, 5864. (f) Bhunia, S.; Ghosh, S.; Dey, D.; Bisai, A. Org. Lett. 2013, 15, 2426.

(2) Zhang, Z.; Liu, Y.; Ling, L.; Li, Y.; Dong, Y.; Gong, M.; Zhao, X.; Zhang, Y.; Wang, J. J. Am. Chem. Soc. 2011, 133, 4330.

(3) Sumino, S.; Fusano, A.; Fukuyama, T.; Ryu, I. Synlett 2012, 23, 1331.

(4) (a) Graziano, M. L.; Cimminiello, G. Synthesis 1989, 54. (b) López-Alvarado, P.; Avendaño, C.; Menéndez, J. C. Tetrahedron Lett. 2001, 42, 4479. (c) Tuba, R.; Ungváry, F. J. Mol. Catal. A: Chem. 2003, 203, 59. (d) Balogh, J.; Kégl, T.; Ungváry, F.; Skoda-Földes, R. Tetrahedron Lett. 2009, 50, 4727.

(5) For reviews, see: (a) Yan, J.; Li, J. H.; Cheng, D. P. Synlett 2007, 2442. (b) Rajender Reddy, K.; Venkateshwar, M.; Uma Maheswari, C.; Santhosh Kumar, P. Tetrahedron Lett. 2010, 51, 2170. (c) Usanov, D. L.; Yamamoto, H. J. Am. Chem. Soc. 2011, 133, 1286. (d) Chen, D. X.; Cao, Y.; Yuan, Z. L.; Cai, H. T.; Zheng, R. W.; Kong, L. C.; Zhu, G. G. J. Org. Chem. 2011, 76, 4071. (e) Li, Y. B.; Liu, X. H.; Jiang, H. F.; Liu, B. F.; Chen, Z. W.; Zhou, P. Angew. Chem. Int. Ed. 2011, 50, 6341. (f) Tang, J. S.; Xiang, X. Y.; Wang, Z. J.; Li, J. H. Synthesis 2011, 2789. (g) Tang, J. S.; Tian, M.; Sheng, W. B.; Guo, C. C. Synthesis 2012, 44, 540. (h) Zeng, W.; Wu, W. Q.; Jiang, H. F.; Sun, Y. D.; Chen, Z. W. Tetrahedron Lett. 2013, 54, 4605.

(6) Perry, A.; Taylor, R. J. K. Chem. Commun. 2009, 3249.

(7) Shair, M. D.; Yoon, T.; Danishefsky, S. J. J. Org. Chem. 1994, 59, 3755.

(8) Mahboobi, S.; Eichhorn, E.; Winkler, M.; Sellmer, A.; Moellmann, U. Eur. J. Med. Chem. 2008, 43, 633.

(9) Chakraborty, K.; Devakumar, C. J. Agric. Food Chem. 2006, 54, 6800.

(10) Manikowski, A.; Kolarska, Z. Synth. Commun. 2009, 39, 3621.

(11) Kuang, Y.; Liu, X.; Chang, L.; Wang, M.; Lin, L.; Feng, X. Org. Lett. 2011, 13, 3814.

This article differs from the e-first online version only in its layout; no content has been changed. 